PROOFS OF SOME INEQUALITIES AND LIMITS.

Therefore the equations of this axis are

$$
x\left(F+f a^{\prime}\right)=y\left(G+g a^{\prime}\right)=\approx\left(H+h a^{\prime}\right) .
$$

Similar equations hold for the other axes, with $b^{\prime}$ and $c^{\prime}$ instead of $a^{\prime}$.

Lawrence Crawford.

\title{
Proofs of some Inequalities and Limits.
}

In his article in No. 20, Professor Gibson gives proofs of the inequalities $1-n a<(1-a)^{n}<\frac{1}{1+n a}$ with certain restrictions as to the values of $n$ and $a$. The mode of proof, aroiding as it does the use of the Binomial Theorem, is comparatively short and simple. The following mode of proof is, I think, still simpler, as it does not involve the use of Mathematical Induction.

If $n$ is a positive integer and $a$ positive, we have

$$
\begin{array}{r}
\frac{(1+a)^{n}-1}{(1+a)-1}=(1+a)^{n-1}+(1+a)^{n-3}+(1+a)^{n-3}+\ldots+(1+a)+1, \\
>n, \quad \\
\therefore \quad(1+a)^{n}-1>n a, \\
\therefore \quad(1+a)^{n}>1+n a . \quad \ldots \ldots \ldots \ldots \ldots \ldots . . .1
\end{array}
$$

Again, $n$ being a positive integer and $a$ a positive proper fraction, we have

$$
\begin{aligned}
& \frac{1-(1-a)^{n}}{1-(1-a)}=1+(1-a)+(1-a)^{2}+\ldots+(1-a)^{n-1}, \\
& <n \text {, } \\
& \therefore \quad 1-(1-a)^{n}<n a \text {, } \\
& \therefore \quad(1-a)^{n}>1-n a \text {. }
\end{aligned}
$$

Then, since $(1-a)(1+a)=1-a^{2}$

$$
\begin{gathered}
<1, \\
\therefore \quad 1-a<\frac{1}{1+a}, \\
\therefore \quad(1-a)^{n}<\frac{1}{(1+a)^{n}}, \\
\therefore \quad \text { by }(1),<\frac{1}{1+n a} .
\end{gathered}
$$


With the further restriction that $n a$ should be a proper fraction, and therefore 1 - na positive, we have

$$
\begin{aligned}
(1+a)^{n} & <\frac{1}{(1-a)^{n}}, \\
\therefore \quad \text { by }(2) & <\frac{1}{1-n a} .
\end{aligned}
$$

Professor Gibson applies these inequalities to prove

$$
\operatorname{Lim}_{n \rightarrow \infty}\left(\cos \frac{x}{n}\right)^{n}=1 \text { and } \operatorname{Lim}_{n \rightarrow \infty}\left\{\left(\sin \frac{x}{n}\right) / \frac{x}{n}\right\}^{n}=1 .
$$

It is perhaps worth noting that (2), or something equivalent to it, is required to establish the formula for the "sum to infinity" of a geometrical progression of which the "common ratio" is a proper fraction.

It is, of course, easy to prove that

$$
c+c r+c r^{2}+\ldots+c r^{n-1}=\frac{c}{1-r}-\frac{c r^{n}}{1-r}
$$

but to deduce the result $\operatorname{Lim}_{n \rightarrow \infty}\left(c+c r+c r^{2}+\ldots+c r^{n-1}\right)=\frac{c}{1-r}$ we must first prove that when $r$ is a proper fraction $\underset{n \rightarrow \infty}{\operatorname{Lim}} r^{n}=0$. In many text-books of algebra this is left unproved without any acknowledgement of the assumption made. It can, of course, be proved easily by the aid of (1) by noting that if $r$ is a proper fraction, we can write

$$
\begin{aligned}
r=\frac{1}{1+a}, \quad \therefore r^{n} & =\frac{1}{(1+a)^{n}}, \\
& <\frac{1}{1+n a},
\end{aligned}
$$

which can obviously be made as small as we please by making $n$ large enough. In fact, if $\epsilon$ is an arbitrarily small positive quantity, we have only to take $n>\frac{1}{\epsilon a}$, and we have $n a>\frac{1}{\epsilon}$, $\therefore \quad 1+n a>\frac{1}{\epsilon}, \therefore \frac{1}{1+n a}<\epsilon$. 
It may be useful to collect here certain other fundamental "limit theorems" which are required for a school course of Algebra, with simple proofs annexed.

(i) If $|x|>1, \underset{n \rightarrow \infty}{\operatorname{Lim} .} x^{n}=\infty$.

Putting $|x|=1+a$, the proof follows immediately from (1), for the case of $n$ a positive integer.

(ii) If $|x|<1, \operatorname{Lim}_{n \rightarrow \infty} x^{n}=0$.

The proof has been given above.

(iii) If $n$ be a positive integer, $\operatorname{Lim}_{n \rightarrow \infty}\left(\frac{x^{n}}{n !}\right)=0$.

First take the case when $n$ is even.

We have

$$
\frac{x^{n}}{n !}=\left(\frac{x}{1} \cdot \frac{x}{n}\right)\left(\frac{x}{2} \cdot \frac{x}{n-1}\right) \cdots\left(\frac{x}{r} \cdot \frac{x}{n-r+1}\right) \cdots\left(\frac{x}{\frac{n}{2}} \cdot \frac{x}{\frac{n}{2}+1}\right) .
$$

Now $r(n-r+1)=(r-1)(n-r)+n$,

$$
\Varangle n \text {, }
$$

the symbol $\varangle$ reducing to $>$ except when $r=1$.

$$
\therefore \frac{x^{n}}{n !}<\left(\frac{x^{2}}{n}\right)^{\frac{n}{2}} \text {. }
$$

Now if $\epsilon$ is an arbitrarily small positive proper fraction, and if $n$ is any integer greater than $\frac{x^{2}}{\epsilon}$, we have $\frac{x^{2}}{n}<\epsilon, \therefore\left(\frac{x^{2}}{n}\right)^{\frac{n}{2}}<\epsilon$.

Thus, when $n$ is even, $\operatorname{Lim}_{n \rightarrow \infty} \frac{x^{n}}{n !}=0$,

When $n$ is odd, let $n=m+1$, so that $m$ is even.

Then proceeding as before, we get

$$
\begin{aligned}
\frac{x^{n}}{n !}=\frac{x^{m}}{m !} \cdot \frac{x}{m+1}, & \\
<\left(\frac{x^{2}}{m}\right)^{\frac{m}{2}} \cdot \frac{x}{m+1} & <\left(\frac{x^{2}}{m}\right)^{\frac{m}{2}} \cdot \frac{x}{\sqrt{m}}, \\
& <\left(\frac{x^{2}}{m}\right)^{\frac{m+1}{2}},
\end{aligned}
$$

and this, as before, has zero as its limit. 
Corollary. $n !>n^{\frac{n}{2}}$,

(iv) $\operatorname{Lim.}_{h \rightarrow 0}\left(a^{h}-1\right)=0$, or $\operatorname{Lim}_{h \rightarrow 0} a^{h}=1$.

Put $h=\frac{1}{n}$, when $n$ is a positive integer, so that $n \rightarrow \infty$ when $h \rightarrow 0$.

First suppose $a$ positive and greater than 1 .

Then $a^{\frac{1}{n}}-1=\frac{a-1}{a^{\frac{n-1}{n}}+a^{\frac{n-2}{n}}+\ldots+a^{\frac{1}{n}}+1}$,

$$
<\frac{a-1}{n} \text {. }
$$

Hence Lim. $\left(a^{\frac{1}{n}}-1\right)=0$.

$$
n \rightarrow \infty
$$

Next suppose $a$ to be a positive proper fraction.

Then

$$
\begin{aligned}
1-a^{\frac{1}{n}}=\frac{1-a}{1+a^{\frac{1}{n}}+a^{\frac{2}{n}}+\ldots+a^{\frac{n-1}{n}}} & =\frac{a^{-1}-1}{a^{-1}+a^{-\frac{n-1}{n}}+a^{-\frac{n-2}{n}}+\cdots+a^{-\frac{1}{n}}} \\
& <\frac{a^{-1}-1}{n} .
\end{aligned}
$$

Hence $\operatorname{Lim}_{n \rightarrow \infty}\left(1-a^{\frac{1}{n}}\right)=0$.

The restriction that $n$ should tend to infinity by integral values is, as pointed out in Professor Gibson's last paragraph, easily removed.

Thus, $\underset{h \rightarrow 0}{\text { Lim. }} a^{h}=1$.

Corollary. $\quad a^{x}$ is a continuous function of $x$.

For $a^{x+h}-a^{x}=a^{x}\left(a^{h}-1\right)$,

$\therefore$ Lim. $\left(a^{x+h}-a^{x}\right)=0$, since $a^{x}$ is finite, $h \rightarrow 0$

$\therefore \quad$ Lim. $a^{x+h}=a^{x}$, $h \rightarrow 0$

$\therefore \quad a^{x}$ is a continuous function of $x$.

R. F. Muirhead. 